\title{
Frequency Estimation Using SAMP-SVD Based on Nyquist Folding Receiver
}

\author{
Jun Wang , Sujuan Chen , Kaili Jiang , and Bin Tang \\ School of Electronic Engineering, University of Electronic Science and Technology of China, Chengdu, Sichuan, P.R. China
}

\begin{abstract}
The Nyquist Folding Receiver (NYFR) is an efficient analogy-to-information (A2I) architecture that folds the broadband RF inputs by subsampling so that can sample with a low-speed ADC. The compressive sensing (CS) model of the NYFR can be built in the sparse environment to recover by traditional CS algorithms. This paper presents an improved algorithm SAMP-SVD based on sparsity adaptive matching pursuit (SAMP) and singular value decomposition (SVD) which is intended to deal with the problem caused by SAMP recovering - the inversion of matrix, avoiding the problem of whether matrix is singular and reducing the time of computation. A comparative analysis between the presented method and sparse reconstruction by separable approximation (SpaRSA) is discussed; simulation results show the algorithm can reconstruct the signal effectively and estimate the frequencies accurately.
\end{abstract}

\section{Introduction}

The bottleneck in reconnaissance receiver performance is in the analog-to-digital conversion (ADC) process, where sampling rate and accuracy in limitation [1], cannot matching to radar coverage. Given this discord, we need to improve the receiving system on the present device level, breaking the limitation of ADC.

There are currently several sub-sampling architectures: the random demodulator (RD) [2] and the modulated wideband converter (MWC) [3] as a practical implementation of compressive sensing (CS) [4], where the signals is sampled completely randomly for suppression of aliasing and guaranteed reconstruction. Another efficient analogy-to-information (A2I) architecture is called the NYFR [5] in 2006 presented, which folds the broadband radio frequency (RF) input by sub-sampling with a stream of short pulses. The modulation sampling induces a bandwidth widening that varies with the Nyquist zone (NZ, i.e. folding index number). It is a periodic non-uniform sampling, which is a tradeoff between folding and aliasing. The undersampled signals then digitized by a narrowband ADC, which the same as CS techniques by using only a few non-zero coefficients in some suitable sparse representation, allowing recovery techniques in addition to traditional CS recovery methods such as reconstruction via $l_{1}$-minimization [6].
There are a number of classical approaches to recover information from signals, which are obtained as the solution to the constrained minimization problem in a CS setting, including $l_{1}$-minimization, Sparse Reconstruction by separable approximation (SpaRSA) [7], iterative greedy unfolding (IGU) [8] and matching pursuit (MP) [9], etc. $l_{1}$-minimization and SpaRSA are by means of optimization theory, the former tend to be slow for extremely large sampled data sets and, ill-suited for nonpositive definite measurement matrix, if speed is not an issue, SpaRSA obtains higher resolution accuracy. MP and orthogonal matching pursuit (OMP) [10] techniques are the basis methods on pursuit algorithms, which enjoy highly efficient, low computational complexity and a restricted isometry property (RIP) [11]. stagewise orthogonal matching pursuit (StOMP) [12] reduces the complexity of the algorithm, but its accuracy not as good as OMP. regularized orthogonal matching pursuit (ROMP) [13] and compressive sampling matching pursuit (CoSaMP) [14] require the sparsity knowledge of signals. subspace pursuit (SP) [15] algorithm performance is similar to CoSaMP, and combination with OMP to get a powerful methods named SAMP [16].

This paper focuses on SAMP because of its efficiency and ease of implementation, and applies SVD to solve the least squares (LS) problem, for a high-accuracy frequency estimation under the condition of a low signalto-noise ratio (SNR). 


\section{NYFR Sensing Matrix Model}

CS builds upon the fundamental fact that signals can be represented by using only a few non-zero coefficients in a suitable basis or dictionary, projecting a highdimensional signal onto a low-dimensional signal model, recovered such signals from very few measurements by optimization algorithm, to break through the traditional limits of sampling theory.

Assumptions about the analog RF input signals $x_{a}(t)$ of the NYFR architecture in a usual CS setting [8]; namely:

1) the input $x_{a}(t)$ can be sampled according to the

Nyquist theorem, denoted as $x[n]$.

2) the discrete Fourier transform (DFT) $X[k]$ of $x[n]$

is sparse or highly compressible.

Note that the relationship between the NYFR architecture and the theory of CS can be presented as following in the assumptions setting. Then we represent $X[k] \in \mathbb{C}^{N}$ as a vector of s-sparse (i.e., the number of non-zero elements is $s$ in the frequency domain), and consider the output measurement vector $y[n] \in \mathbb{C}^{K}$ after ADC sampling. Expressed the NYFR receiving model by underdetermined linear equations

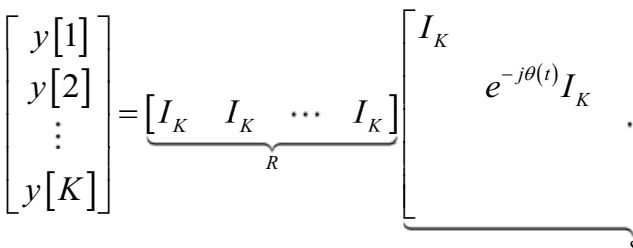

Given the sensing matrix model without the estimation of folding index number as the reference [8]. The DFT of Nyquist rate sampled inputs with the length of $N$, partitioned into $Z$ index numbers of length $K$ each block matrix, meets $N=K \cdot Z$. Then induced modulation by diagonal matrix $S \in \mathbb{C}^{N \times N}$ which is the function of time on the inverse DFT (IDFT) block matrices $\Psi \in \mathbb{C}^{N \times N}$ of inputs with index numbers from zero to $Z$ and, projected into baseband of length $K$ also with matrix $R \in \mathbb{C}^{K \times N}$, where $R, S$ and $\Psi$ are partitioned as $X[k]$.This model is depicted by

where the measurement matrix is $\phi=R S \in \mathbb{C}^{K \times N}$, and the sensing matrix is $\Phi=R S \Psi \in \mathbb{C}^{K \times N}$, the block matrix of $X[k]$ is given by

$$
X_{i}[k]=\left[\begin{array}{c}
X[(i-1) K+1] \\
X[(i-1) K+2] \\
\vdots \\
X[i K]
\end{array}\right] \in C^{K \times 1}, i=1, \ldots, Z
$$

and the IDFT matrix of length $K$ is

$$
y[n]=\phi x[n] \text { or } y[n]=\Phi X[k]
$$

where $\phi \in \mathbb{C}^{K \times N}$ is measurement matrix and, $\Phi \in \mathbb{C}^{K \times N}$ is sensing matrix. Note that $N=K \cdot Z$, where $Z$ denotes index number (or folding number).

Motivated by RD model, the direct measurement matrix (D-measurement matrix) is given by

$$
\phi=\operatorname{diag}\left(P_{1}, P_{2}, \cdots, P_{K}\right)
$$

where $P_{i}=\left[p_{1+(i-1) Z}, p_{2+(i-1) Z}, \cdots, p_{Z+(i-1) Z}\right], i=1,2, \cdots, K$, and $p_{j}=\exp \left(-j\left(2 \pi k f_{s 1} t_{j}+k_{H} \theta\left(t_{j}\right)\right)\right), j=1,2, \cdots, N$, namely that the modulation sampling function is sampled by the Nyquist rate of the input $x_{a}(t)$.

A next consideration is signal reconstruction from $y[n]$ and $\phi$ by CS techniques. Actually, the NYFR signal reconstruction is dependent of $k_{H}$ (namely, folding index number) estimated correctly and the synchronization of measurement matrix sampled with wideband-input signals under Nyquist theorem, from the mathematical point-of-view. Thus the sensitivity of folding index number estimation and the synchronization of sampling with inputs are important to estimate accurately, which show the deficiencies.

$\left.e^{-j(Z-1) \theta(t)} I_{K}\right] \underbrace{\left[\begin{array}{llll}\Psi_{K} & & & \\ & \Psi_{K} & & \\ & & \ddots & \\ & & & \Psi_{K}\end{array}\right]}_{\Psi}\left[\begin{array}{c}X_{1}[k] \\ X_{2}[k] \\ \vdots \\ X_{Z}[k]\end{array}\right]$

$$
\Psi_{K}=\frac{1}{\sqrt{K}}\left[\begin{array}{ccccc}
1 & 1 & 1 & & 1 \\
1 & \omega^{1} & \omega^{2} & \cdots & \omega^{K-1} \\
1 & \omega^{2} & \omega^{4} & \cdots & \omega^{2(K-1)} \\
\cdots & \cdots & \cdots & \cdots & \cdots \\
1 & \omega^{K-1} & \omega^{2(K-1)} & \cdots & \omega^{(K-1)(K-1)}
\end{array}\right]
$$

where $\omega=e^{j 2 \pi / K}$. Nevertheless the deficiency on synchronization based on this model is the same as the model mentioned before, from the practical consideration.

\section{Information Recovery}

The OMP and the SP are the typical greedy algorithm of the CS algorithm, which can search for answers in the several regions of a solution space and jump out of local optimization at a greater probability, thus the global optimization can be found. The SAMP is the combination of the OMP look-ahead searching and the SP backtracking searching, modifying the support set (SS) with a fixed step length in iterative process, eliminating the poor atoms via new and the backtracking searching until residuals no longer shrinking.

The SAMP brings solution for blind signal recovery which does not require information of sparsity $s$ of input signals as a prior, following greedy iteration principles 
through stage by stage to estimates the sparsity. The OMP and the SP are viewed as its special cases, namely that when $\hat{s}=1$, SAMP can be roughly regarded as OMP which takes a few more iterations on more accuracy. In addition, when $\hat{s}=s$, SAMP becomes exactly SP. Thus, the stage is generally chosen one from $\hat{s}=1 \sim s$. The improved SAMP algorithms which applied SVD on the basis of previous study for high-resolution reconstruction, called SAMP-SVD.

The following Table I shows the steps of SAMP algorithm [16].

Table 1. SAMP Steps

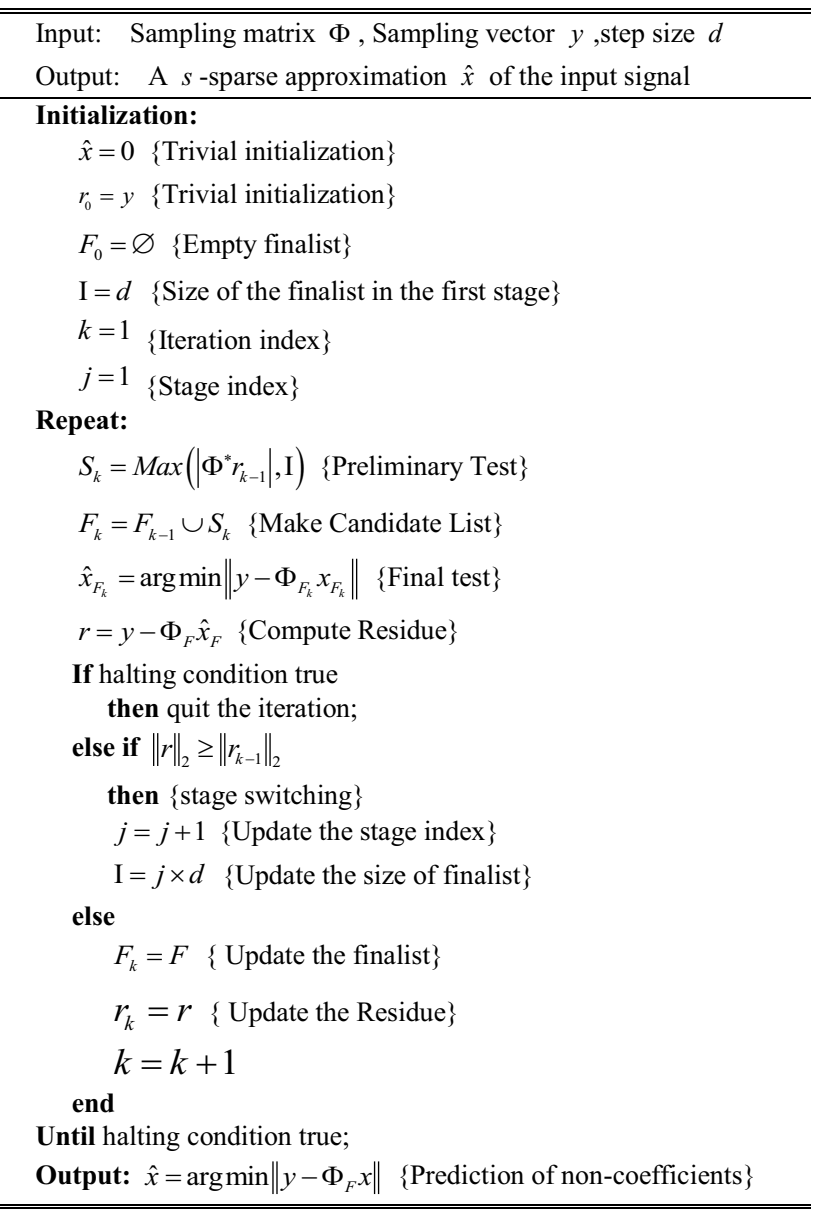

An optimum solution is found by the minimum of residual to $\hat{x}_{F_{k}}=\arg \min \left\|y-\Phi_{F_{k}} x_{F_{k}}\right\|$, which is equivalent to a least squares problem as $\left(\Phi_{F_{k}}^{H} \Phi_{F_{k}}\right) x_{F_{k}}=\Phi_{F_{k}}^{H} y$ and, namely $\hat{x}_{F_{k}}=\left(\Phi_{F_{k}}^{H} \Phi_{F_{k}}\right)^{-1} \Phi_{F_{k}}^{H} y$. However, in solving the normal equation we are interested in the singularity of $\Phi_{F_{k}}^{H} \Phi_{F_{k}}$, unfortunately, the inverse of a nonsingular matrix costs a large computation and is difficult to implement in computer arithmetic. The SVD definitely point out the generalized inverse numerical solution of $\Phi_{F_{k}}^{H} \Phi_{F_{k}}$ which is avoided in the problem of singularity.
The solution of LS regular system by using SVD algorithm

$$
\hat{x}_{F_{k}}=\sum_{i=1}^{l} u_{i}\left(u_{i}^{H} \Phi_{F_{k}}^{H} y / \sigma_{i}^{2}\right)
$$

where $u_{i}$ is the right singular vector, $\sigma_{i}$ represents nonzero singular value and, the number of singular values is $l$ which depends on $\Phi_{F_{k}}^{H} \Phi_{F_{k}}$.

And the following Table II shows the steps of SVD decomposition.

Table 2. SVD Decomposition Steps

Step 1: the singularly valuable decomposition of $\Phi_{F_{k}}$ all the nonzero singular value is $\sigma_{1}, \sigma_{2} \cdots \sigma_{s}$ the corresponding right singular vector $u_{1}, u_{2} \cdots u_{s}$

Step 2: output the numerical solution $\hat{x}_{F_{k}}=\sum_{i=1}^{l} u_{i}\left(u_{i}^{H} \Phi_{F_{k}}^{H} y / \sigma_{i}^{2}\right)$

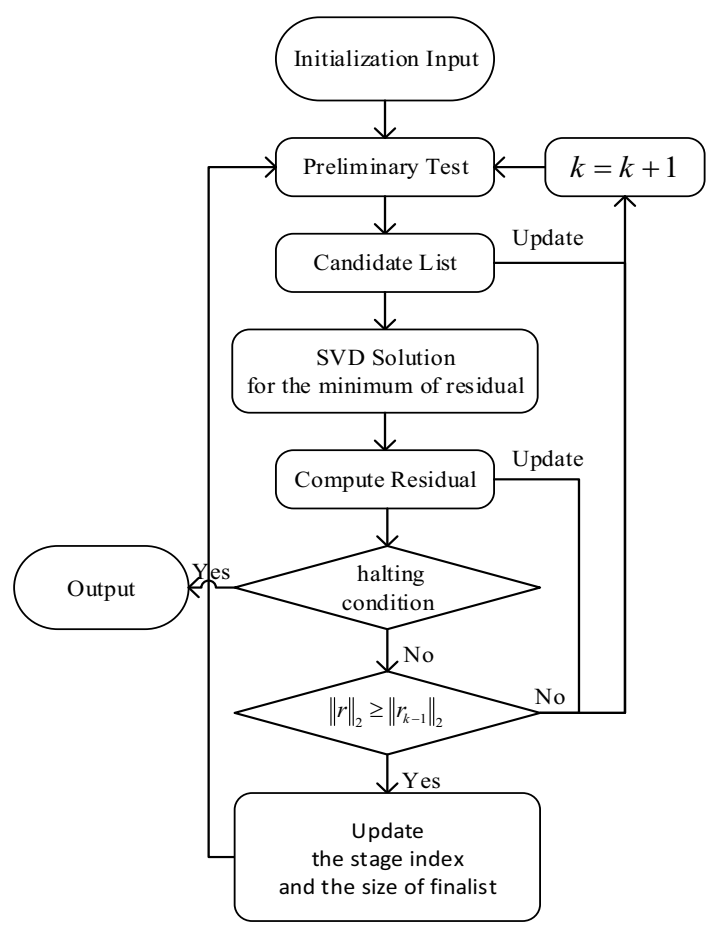

Fig. 1 The Flow Chart of SAMP-SVD Algorithm

where the output gets the numerical solutions from SVD algorithm to avoid the complexities of matrix inversion. SAMP-SVD obtains high-resolution reconstruction by combining the merits of SAMP and SVD, not requiring sparsity and singularity as prior. The flow chart of the SAMP-SVD algorithm is presented in Fig.1.

\section{Results and Discussion}

In this section, the performance of SAMP-SVD algorithm derived above is evaluated through simulation and compared with the SpaRSA that is one of traditional CS convex recovery algorithm, and we briefly discuss 
their strengths and weaknesses. The inputs can be written as fellows shown in (7)

$$
x(n)=\sum_{\mathrm{m}=1}^{N} A_{m} \cos \left(2 \pi f_{m} n+\varphi_{m}\right)+w(n)
$$

where the $A_{m}$ is the amplitude of the $m$ th signal, $f_{m}$ and $\varphi_{m}$ represent the frequency and phase of input signal, and $w(n)$ is a vector of Gaussian white noise.

Example 1: At the first experiment, we will show the information recovery performance of the two matrices mentioned above. We consider the parameters of input signals are $f=5.36 \mathrm{GHz}, A=1, \varphi=\pi / 6$, and assume the environment is without noise. For measurement matrix $\phi$, we choose average sampling rate $f_{s 1}$ of $2 \mathrm{GHz}$, and the modulated function $\theta(n)$ is written as $\sin \left(2 \pi f_{\theta} n\right)$. As discussed earlier, in order to reconstruct more highly accuracy, we should give a true index zones. The NYFR sensing matrix $\Phi$ has the same modulated function as $\phi$. Fig. 2 shows the SAMP-SVD reconstruction spectrum obtained from both of two. Notice that there is an extra frequency at $6.65 \mathrm{GHz}$ in the first picture which use Dmeasurement matrix, but it is pure in the spectrum of second. As a consequence, we will use the NYFR sensing matrix in the following experiments.
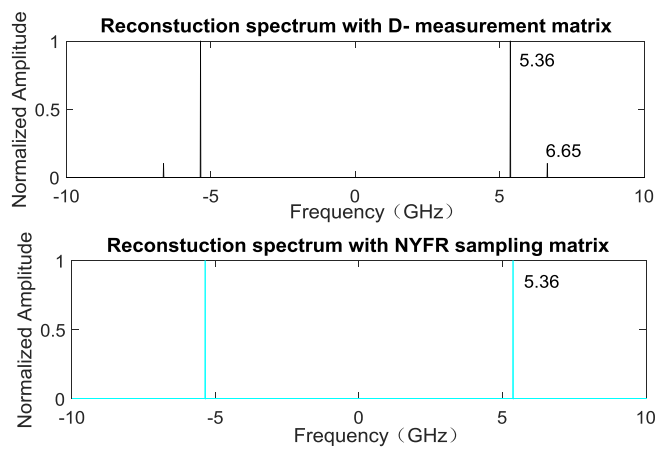

Fig. 2 Spectrum of reconstruction signal

Example 2: In this experiment, we assume that the input of NYFR consisting of monopulse (MP), linear frequency modulation (LFM) and binary phase shift keying (BPSK) signals at 2.1, 5.56, and 12.94GHz, and consider the amplitude of each signals are 1 , the phase are $\pi / 6, \pi / 3$ and $\pi / 2$, respectively. LFM signal is given a fixed-bandwidth of $5 \mathrm{MHz}$, for the BPSK signal case, we choose random codes. Besides, the relative SNR is $10 \mathrm{~dB}$. Fig. 3 shows the SAMP-SVD reconstruction magnitude spectrum of three input signals, in which algorithm uses the NYFR sensing matrix $\Phi$. Notice that the modulated function $\theta(n)$ is written as $\sin \left(2 \pi f_{\theta} n\right)$, where $f_{\theta}$ is $10 \mathrm{MHz}$.

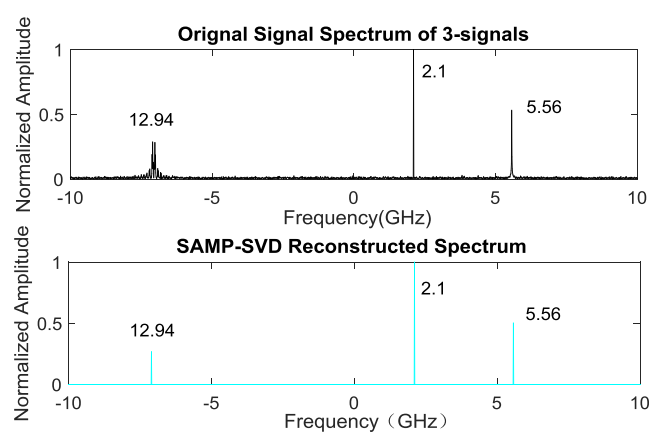

Fig. 3 Spectral of original and SAMP-SVD reconstruction signal

Comparing the spectrum of reconstruction signals with one of the original signals, we can see that SAMP-SVD algorithm recovers the 3 -signal after the NYFR correctly. The accuracy of reconstruction is controlled by noise energy $\sigma^{2}$, comparing with residue $r$ which is updated in each iteration, repeating this step until $r \leq \sigma^{2}$. It can estimate all the frequencies by search for the peaks in the spectrum.

Example 3: Here we consider the situation almost the same with example 1, and we define normalized root mean square error (NRMSE) to evaluate the performance of the frequency estimation by using SpaRSA and SAMP-SVD, the results are based on 500 Monte Carlo simulations and shown in Fig.4, respectively.

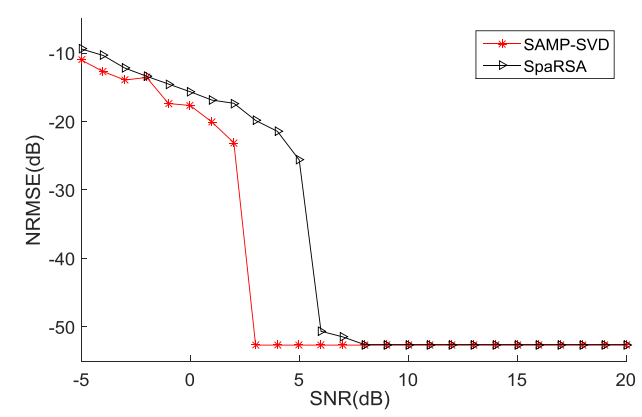

Fig. 4 NRMSE of frequency estimation (SAMP-SVD \& SpaRSA)

The simulation shows the ability of the proposed algorithm on information reconstruction of inputs more faster than SpaRSA, because of SAMP-SVD without the process of matrix inversion, if time is a factor should be considered, the SAMP-SVD is a better choice. Fig.4 shows the NRMSE tends to a certain value when the SNR is greater than $8 \mathrm{~dB}$, and the performance of two algorithm is close to each other.

\section{Conclusions}

SAMP-SVD exhibits a tradeoff in computational complexity versus reconstruction performance if the RIP condition of measurement matrix is satisfied, contributes that not requiring sparsity of the receiving signals and singularity of sensing matrix as prior. And simulation 
shows that the algorithm achieves accurate frequency estimation than the existing SpaRSA in the low SNR environment. Future work will concentrate on wideband signals recovery and the effect of RIP on signal recovery based on the NYFR with information recovery methods of CS framework, promising for selecting the parameters and improving the structure of the NYFR.

\section{References}

1. R. H. Walden. "Analog-to-digital converter survey and analysis," IEEE Journal on Selected Areas in Communications, vol. 17(4), pp. 539-550, Apr 1999.

2. S. Kirolos, J. Laska, M. Wakin etc. "Analog-toInformation Conversion via Random Demodulation[C]," Processing of the IEEE Dallas Circuits and Systems Workshop (DCAS). Dallas, Texas, 2006.

3. J. Laska, S. Kirolos, Y. Massoud etc. Random Sampling for Analog-to-Information Conversion of Wideband Signals[C]. Proceedings of the IEEE Dallas Circuits and Systems Workshop (DCAS'06). Dallas, Texas, 2006.

4. Cand'es, J. Romberg, and T. Tao, "Robust uncertainty principles: Exact signal reconstruction from highly incomplete frequency information," IEEE Trans. on Information Theory, vol. 52, pp. 489-509, Feb 2006.

5. G. Fudge, R. Bland, M. Chivers, S. Ravindran, J. Haupt, and P.Pace, "A Nyquist folding analog-toinformation receiver," in Proc. 42nd Asilomar Conf. on Signals, Compute, Syst. (ACSSC), 2008, pp.541545E.

6. J. Haupt and R. Nowak, "Signal reconstruction from noisy random projections," IEEE Trans. Inf. Theory, vol. 52, no. 9, pp. 4036-4048, Sept.2006.

7. S. J. Wright, R. D. Nowak, and M. A. T. Figueiredo, "Sparse reconstruction by separable approximation,"
IEEE Trans. Signal Process., vol. 57, no. 7, pp. 2479-2493, Jul 2009.

8. R. Maleh, G. L. Fudge, F. A. Boyle, P. E. Pace, et al. "Analog-to-Information and the Nyquist Folding Receiver," IEEE Journal on Emerging and Selected in Circuits and Systems, 2012,2(3) . pp. 564-578.

9. S. G. Mallat and Z. Zhang, "Matching pursuits with time-frequency dictionaries," IEEE Trans. Signal Process., vol. 41, no. 12, pp.3397-3415, Dec 1993.

10. Y. Pati, R. Rezaiifar, and P. Krishnaprasad, "Orthogonal matching pursuit: Recursive function approximation with application to wavelet decomposition," in Proc. 27th ACSSC, 1994, vol. 1, pp. $40-44$.

11. Candes. E. J and Tao. T, "Decoding by linear programming [J]," IEEE Transactions on Information Theory, vol. 51, pp. 4203-4215, Dec 2005.

12. Donoho D. L, Tsaig. Y, Drori. I, et al. "Sparse solution of underdetermined linear equations by stage wise orthogonal matching pursuit [J]," IEEE Transaction on Information Theory, vol. 58, pp. 1094-1121, Feb 2012.

13. D. Needell and R. Vershynin. "Uniform uncertainty principle and signal recovery via regularized orthogonal matching pursuit," Foundations of Computational Mathematies, vol. 9, pp. 317-334, Mar 2009.

14. D. Needell, J. A. Tropp. "CosaMP: Iiterative Signal Recovery from Incomplete and Inaccurate Samples[J]," Applied and Computational Harmonic Analysis, vol. 26, pp. 301-320, Mar 2008.

15. Dai. W, Milenkovic. O, "Subspace pursuit for compressive sensing signal reconstruction," IEEE Trans. Information Theory, vol.55, pp. 2230-2249, May 2009.

16. Thong T D, Gan Lu, Nguyen. "Sparsity adaptive matching pursuit algorithm for practical compressed sensing [J]," in Proc. 2008 42nd Asilomar Conf, 2008, pp. 581-587. 